\title{
Resolution enhancement for geoelectrical layer interpretation of electrical resistivity model from composite dataset: implication from physical model studies
}

\author{
Rajesh Rekapalli*, Dewashish Kumar and V. S. Sarma \\ CSIR-National Geophysical Research Institute, Hyderabad 500 007, India
}

Besides the high resolution offered by HERT, the resistivity models obtained using different electrode arrays differ significantly in geological structure and resistivity range. We combine the apparent resistivity data from multiple arrays to provide single resistivity model of high resolution as 'composite method'. Initially, the method was tested on physical model data obtained over horizontal marble sheet kept in water. The parameters of target (resistivity, geometry, thickness) noticed in the resistivity model corresponding to composite method are appropriately matching with the true parameters. Finally, the method was applied to the data from Mahabubnagar, Telangana, India for groundwater studies. The resistivity model obtained using the proposed method has shown good match with regional hydro-geology and borehole data. The results from physical model as well as field data suggest plausible resolution enhancement in composite methodology for resolving thin layer(s) in 2D and 3D electrical resistivity tomography and induced polarization (IP) studies.

Keywords: Electrical resistivity tomography, groundwater, induced polarization, physical model studies, resolution enhancement.

DEVELOPMENT of resistivity methods, which has been very rapid in the last three decades through the initiation of super-fast computers in data acquisition and processing, has increased their usage in geophysical stu$\operatorname{dies}^{1-3}$. High-resolution electrical resistivity tomography (HERT) is a high-end technology in the resistivity method, which provides both lateral and depth-wise information of the subsurface geological features in a $2 \mathrm{D}$ cross-section $^{4-6}$. Over the past one decade, HERT has been successful in providing significant results in the investigation of 2D and 3D features of subsurface geological formations.

Although HERT is an advanced technique, the electrode arrays employed in the field procedure are the same

*For correspondence. (e-mail: rekapalli@gmail.com) as those in conventional arrays ${ }^{7,8}$. The sensitivity and resolution contrast between the electrode arrays play a major role in the selection of optimum electrode arrays for a specific geophysical investigation ${ }^{4}$. Researchers have also compared different electrode arrays using laboratory models and field studies ${ }^{9-23}$ to understand the depth of investigation and sensitivities for their optimal field operation. Accordingly, dipole-dipole array is more sensitive to laterally extended formations, whereas the Schlumberger array is preferable for vertically extended geological formations. Thus, the selection of a proper array is necessary for accurately mapping the geological features of a region. As the subsurface formations show variations in their lateral and depth extents, it is not possible to estimate the parameters of the formation precisely using a single electrode array.

In multi-electrode resistivity surveys, high resolution can be achieved using dipole-dipole array with small inter-electrode spacing. However, smaller inter-electrode spacing reduces the depth of investigation ${ }^{16,24,25}$. In general, Schlumberger array is more suitable for vertical electrical sounding because of its geometrical convenience for field operations. The Wenner-Schlumberger configuration - a hybrid configuration of conventional Wenner and Schlumberger arrays, is used to map lateral and vertical extensions of subsurface objects with moderate resolution $^{4}$. In addition, increasing the resolution by altering the electrode separation will have an inverse effect on depth of investigation ${ }^{25}$, and vice versa. The pitfalls of resistivity imaging in connection with resolution have already been reported ${ }^{26}$. Apart from the inversion method, a probability-based approach has also been used in $2 \mathrm{D}$ imaging to address the uncertainties in interpretation to certain extent ${ }^{27-29}$.

We noticed considerable mismatch among crosssection images obtained using dipole-dipole, Wenner, Schlumberger and Wenner-Schlumberger arrays, both with regard to geological structure and resistivity range along the same profile with the same profile constants (i.e. inter-electrode spacing and profile length). This causes diversity or ambiguity in interpretation while 


\section{RESEARCH ARTICLES}

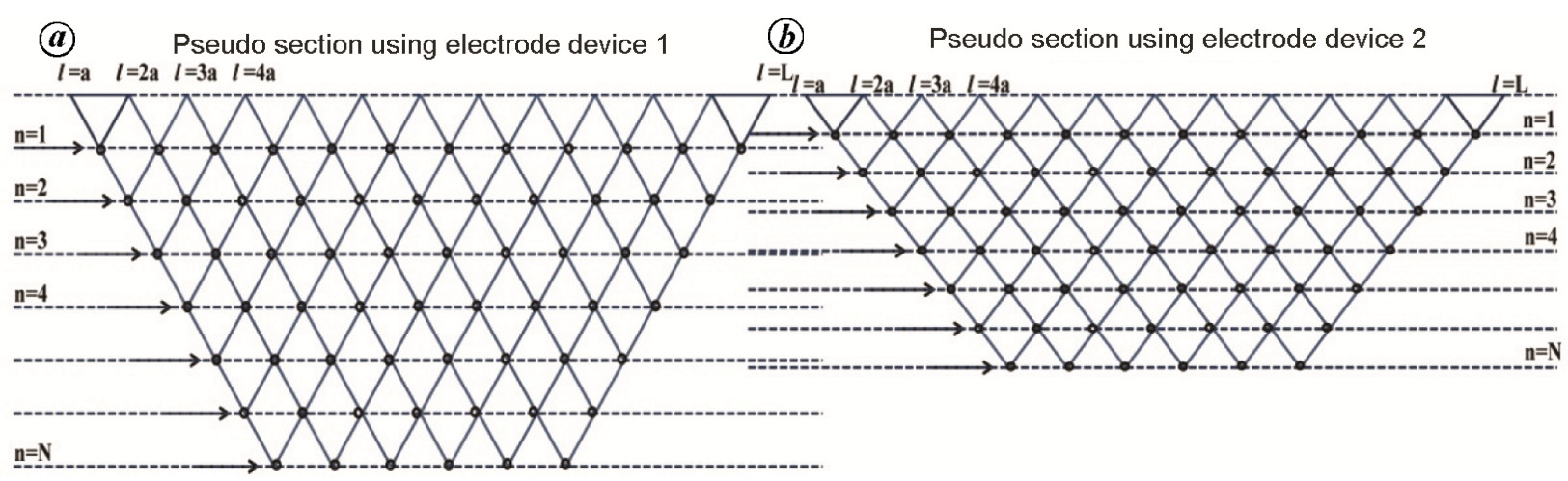

Figure 1. Pseudo depth section of two different electrode arrays (devices).

using the cross-section images of individual configurations.

The ambiguity is the result of difference among the resolution indices and depths of investigation (or pseudo depths) of different arrays. The data obtained along the same profile using different arrays tend to map different depths in the cross-section/pseudo section for any geological strata. The generation of the cross-section images involves interpolation between the measured data levels. This leads to uncertainty regarding the geological structure and resistivity range observed from cross-section images of different arrays for the same profile. In the present study, we present 'composite methodology' to resolve the aforementioned ambiguity by integrating the data from different arrays to produce only one crosssection image where the resolution is enhanced by minimizing the interpolation. First we present the physical model laboratory experiment over a known marble sheet (length $=60 \mathrm{~cm}, \quad$ breadth $=30 \mathrm{~cm}, \quad$ thickness $=2 \mathrm{~cm})$ immersed in water inside a non-conducting tank. Then we apply the method to field data from Mahabubnagar district, Telangana, India, for interpreting the groundwater potential zones and assessing recharge characteristics using resistivity and induced polarization (IP) data.

\section{Methodology}

Theoretically, investigation depth of any electrode array is constant for a fixed set of profile constants within a region ${ }^{16,17,30}$. Different electrode arrays possess different investigation depths for the same profile constants. Due to difference in the depth of investigation of different electrode arrays, depth to the data levels varies and thereby produces ambiguity both in the structure and resistivity range for a given geological set-up. For example, consider apparent resistivity data $\rho_{o}(l, n)$ (where $l=1,2, \ldots, L, L$ is the length of the profile and $n=1,2, \ldots, N, N$ is the depth level corresponding to maximum depth) measured by a electrode device (dipole-dipole, Wenner, WennerSchlumberger, etc.) along a profile located on the free surface of an inhomogeneous, isotropic resistivity structure. Each node in the pseudo section $((l, n)$ in the grid) represents the apparent resistivity (true resistivity in the case of inverse model) measured for a particular location of current and potential electrodes (Figure 1). While generating the $2 \mathrm{D}$ tomogram from the inverse model of the above apparent resistivity data, interpolation will take place between the data levels corresponding to different depth levels. As the depth of investigation of different arrays for the same profile length is different, the maximum depth in the pseudo sections also differs (Figure $1 a$ ). The change in interpolation boundaries thus produces difference in the structure and resistivity range among the cross-section images obtained from different electrode devices. Figure $2 a$ and $b$ shows the cross-section images obtained from a profile using dipole-dipole and Wenner-Schlumberger configurations respectively. One can clearly notice differences in the structure and resistivity range of subsurface formations in the figure. It is difficult to interpret a unique geologically consistent model from these images. In addition, Table 1 shows effective depth levels of dipole-dipole and Wenner-Schlumberger configurations of a 60 -electrode profile with $5 \mathrm{~m}$ interelectrode spacing up to a depth of $20 \mathrm{~m}$. One can notice that the depths to the data levels are clearly different for dipole-dipole and Wenner-Schlumberger arrays (Table 1) and also within a depth of $20 \mathrm{~m}$ there are 15 and 10 data levels in dipole-dipole and Wenner-Schlumberger array methods respectively. This demonstrates the high lateral resolution of dipole-dipole configuration for the same profile length and inter-electrode spacing. One can also notice that there is significant difference between the depth levels of these two array configurations, which could lead to difference in the final cross-section images obtained from the data.

Therefore, we attempt here to merge the data from different electrode arrays to form the composite data with increased data levels. For example, the composite data will have 25 data levels (15 from dipole-dipole and 10 from Wenner-Schlumberger) within the $20 \mathrm{~m}$ depth for the example shown in Table 1. Thus the proposed method 

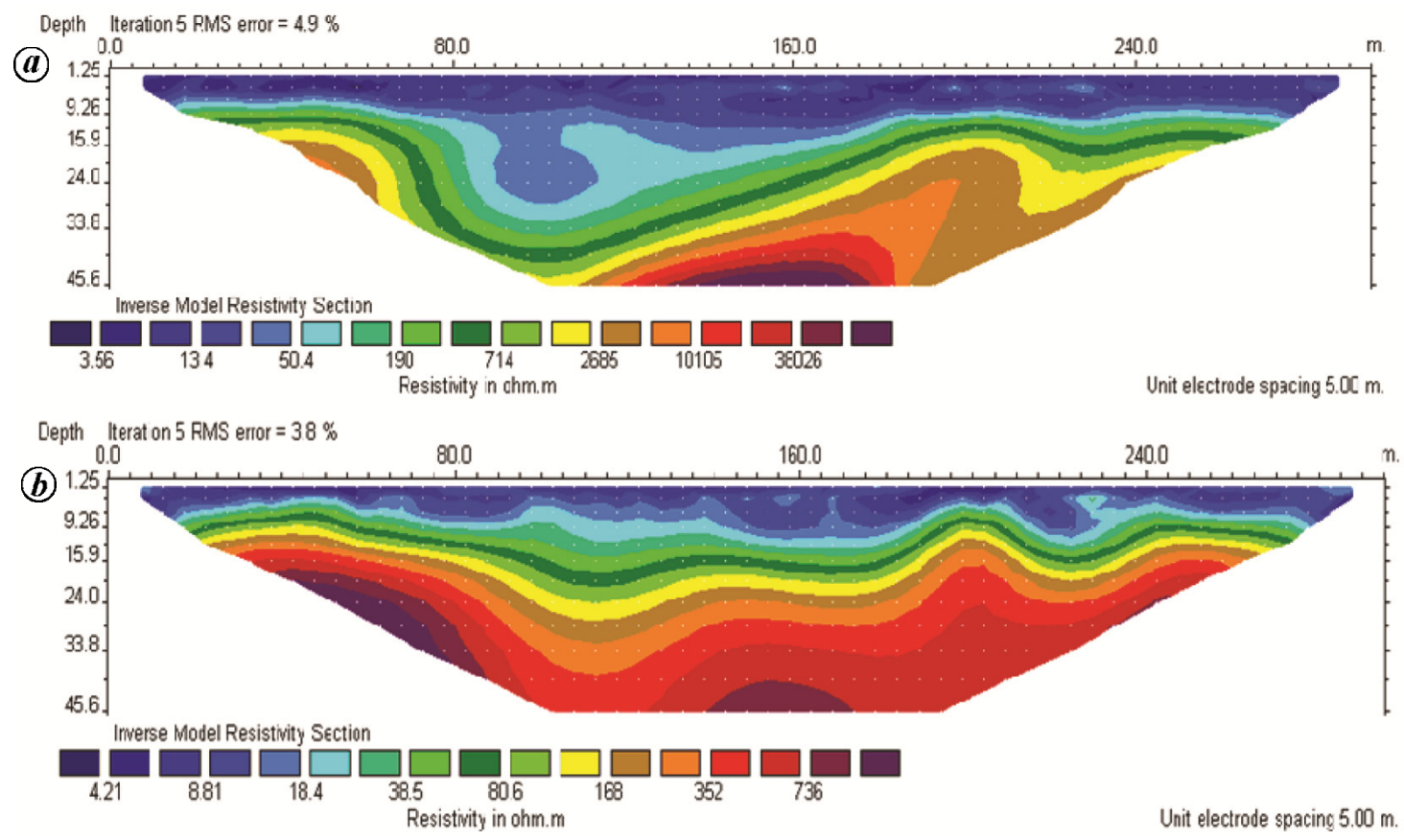

Figure 2. Two-dimensional HERT images of (a) dipole-dipole and (b) Wenner-Schlumberger configurations.

Table 1. Pseudo depth levels of dipole-dipole and WennerSchlumberger arrays

\begin{tabular}{lcc}
\hline & \multicolumn{2}{c}{ Configuration } \\
\cline { 2 - 3 } $\begin{array}{l}\text { No. of depth } \\
\text { levels }(n)\end{array}$ & $\begin{array}{c}\text { Dipole-dipole } \\
\text { Pseudo depth (m) }\end{array}$ & $\begin{array}{c}\text { Wenner-Schlumberger } \\
\text { pseudo depth (m) }\end{array}$ \\
\hline 1 & -2.08 & -2.6 \\
2 & -3.49 & -4.43 \\
3 & -4.81 & -6.59 \\
4 & -6.1 & -8.53 \\
5 & -7.38 & -10.47 \\
6 & -8.65 & -12.39 \\
7 & -9.91 & -14.31 \\
8 & -11.18 & -16.24 \\
9 & -12.44 & -18.16 \\
10 & -13.7 & -20.08 \\
11 & -14.95 & \\
12 & -16.21 & \\
13 & -17.47 & \\
14 & -18.72 & \\
15 & -19.98 & \\
\hline
\end{tabular}

helps to (i) increase the number of data levels that reduces the spacing between the data levels, (ii) minimize interpolation and (iii) obtain single resistivity model for the unique interpretation. This final image obtained from the inversion of the merged data incorporates combined sensitivity and resolves the problems in estimating the subsurface geological features more reasonably from a single electrical tomogram.

As the resolution of HERT depends on the number of apparent resistivity measurements acquired from a unit cube of the subsurface or unit square in the pseudo depth section, the resolution can be increased in the composite cross-section because the number of apparent resistivity measurements is high. Hence the resolution of resistivity image obtained from the composite dataset is higher compared to that obtained from individual configurations. Thus the ambiguity/uncertainty can be resolved with a better resemblance of all the subsurface geological features within one cross section image with enhanced resolution to serve better interpretation. Theoretically, increase in the number of data points within unit depth assures the accuracy of geophysical interpretation of the geo-electrical layers using composite method.

\section{Applications and discussion}

\section{Testing on physical model data}

We tested the proposed phenomenon using physical model experiments. For this, physical model data were acquired in a laboratory model tank ${ }^{31}$ over a known marble sheet (length $=60 \mathrm{~cm}$, breadth $=30 \mathrm{~cm}$, thickness $=2 \mathrm{~cm}$ ) with the Syscal Pro-96 instrument with 48 electrodes with $2 \mathrm{~cm}$ inter-electrode separation. Figure $3 a-c$ shows the cross-section images of dipole-dipole, WennerSchlumberger and composite method over the marble sheet in the physical model tank with tap water as host medium respectively. The cross-section images obtained using dipole-dipole and Wenner-Schlumberger configurations show different target thickness and continuity. The target is not reflected as a sheet of uniform thickness 

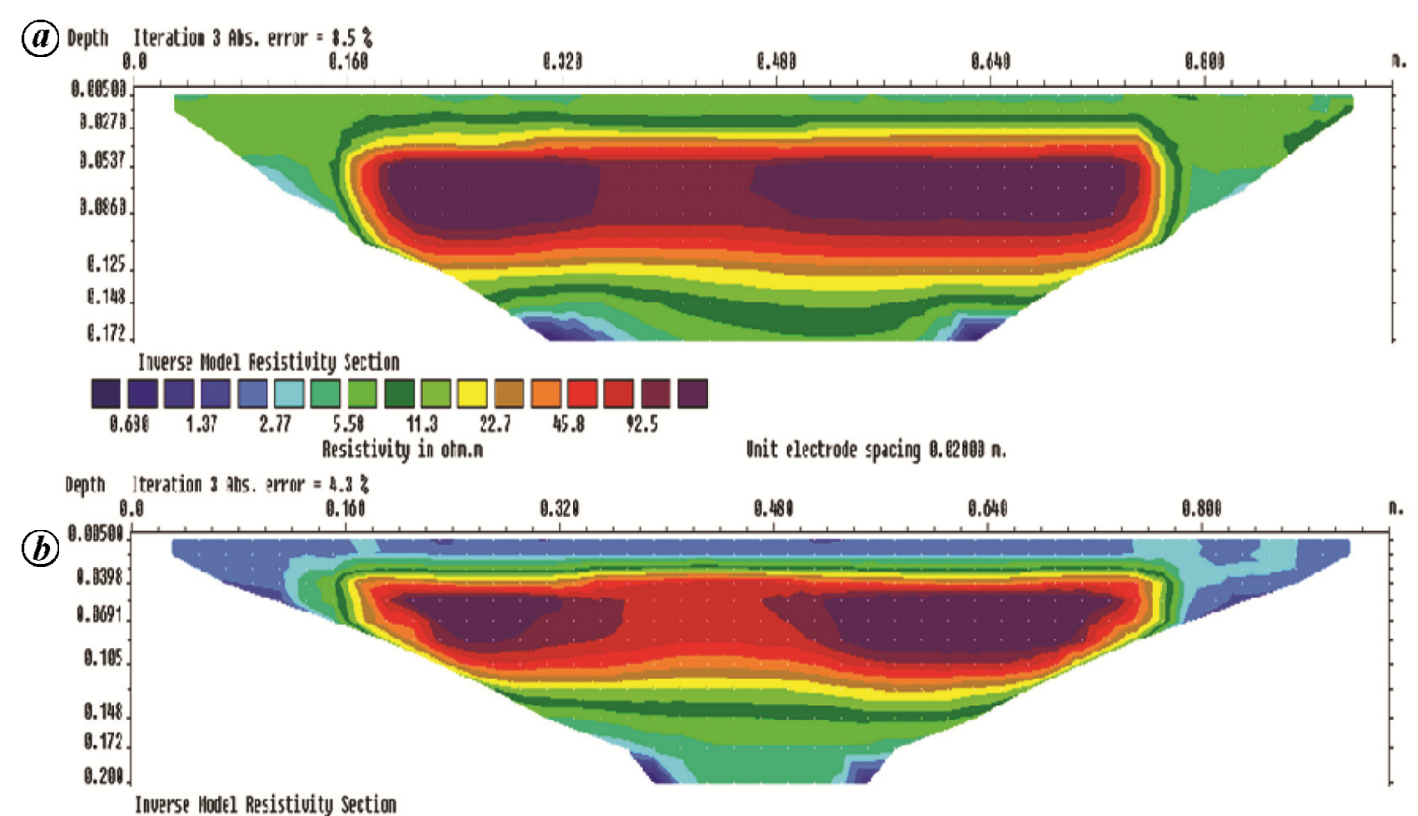

Inverse Hodel Resistivity Section

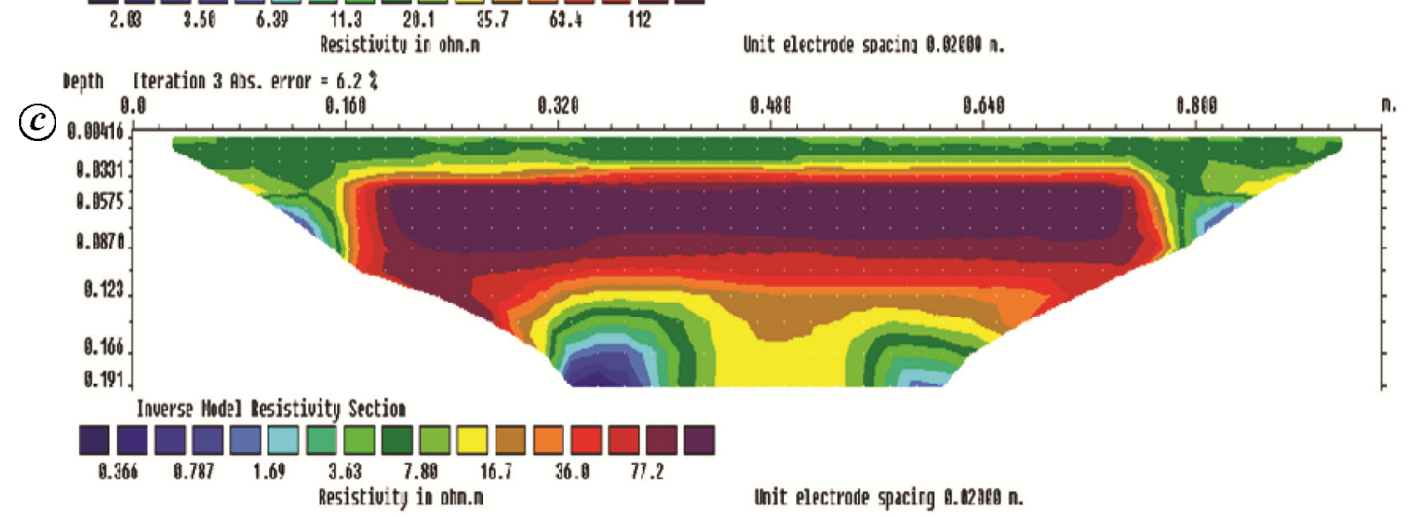

Figure 3. Two-dimensional cross-section images obtained using (a) dipole-dipole, (b) Wenner-Schlumberger and (c) composite arrays in lab physical model over a green marble sheet $(l=60 \mathrm{~cm}, b=30 \mathrm{~cm}, t=2 \mathrm{~cm})$ at depth $d=3 \mathrm{~cm}$.

in the resistivity images of dipole-dipole and WennerSchlumberger configurations. The resistivity of the marble sheet is close to 180 and $210 \mathrm{Ohm} \cdot \mathrm{m}$ in the resistivity models of dipole-dipole and Wenner-Schlumberger arrays respectively. Whereas the cross section obtained from the composite data reflects the resistivity $120 \mathrm{Ohm} \cdot \mathrm{m}$. Although the resistivity of the marble sheet observed in all the three models is close to wet marble resistivity $(100 \mathrm{Ohm} \cdot \mathrm{m})$, the resistivity obtained from composite method is very close to that of the wet marble resistivity. In addition, the marble layer appears as a continuous layer in the model obtained from composite site data. The parameters of the marble layer identified in the model resistivity section corresponding to composite data are more close to the true parameters of the marble sheet used in the modelling. Thus, the results from physical model experiment support the usage of composite array/mixed array for more reliable interpretation of resistivity cross-sections for any $2 \mathrm{D}$ resistivity dataset.

\section{Application to $2 D$ resistivity and induced polarization field data}

The $2 \mathrm{D}$ resistivity and IP data acquired from a granite hard rock terrain in Mahabubnagar district, Telangana, were used here to identify the shallow groundwater pockets based on resistivity and chargeability variations ${ }^{32}$. The data were collected using gradient and dipole-dipole arrays to understand the sub-surface lateral and depthwise heterogeneity in terms of resistivity and chargeability of the geological formation up to a depth $<100 \mathrm{~m}$. Figure $4 a$ and $b$ shows the 2D inverted resistivity models from gradient and dipole-dipole arrays respectively. The resistivity models of gradient array (Figure $4 a$ ) and dipoledipole array (Figure $4 b$ ) show layered structure with a gradual increase in resistivity with depth. However, the resistivity values show clear deviation. The true resistivity values observed from dipole-dipole cross-section image are smaller compared to gradient array. On the other 

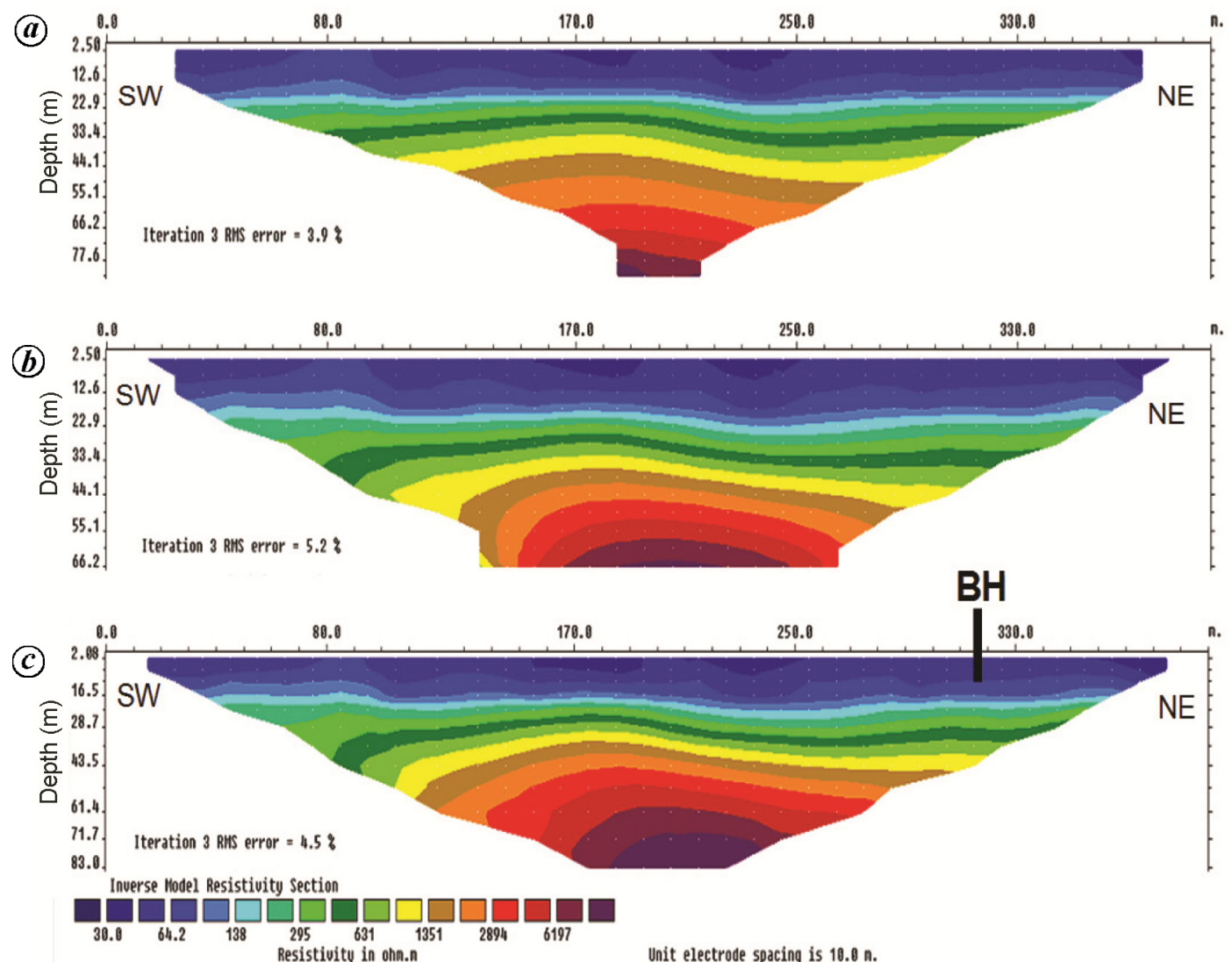

Figure 4. Two-dimensional inverted resistivity model from (a) gradient array data, (b) dipole-dipole array data and (c) composite data in a granitic hard rock area ${ }^{32}$.

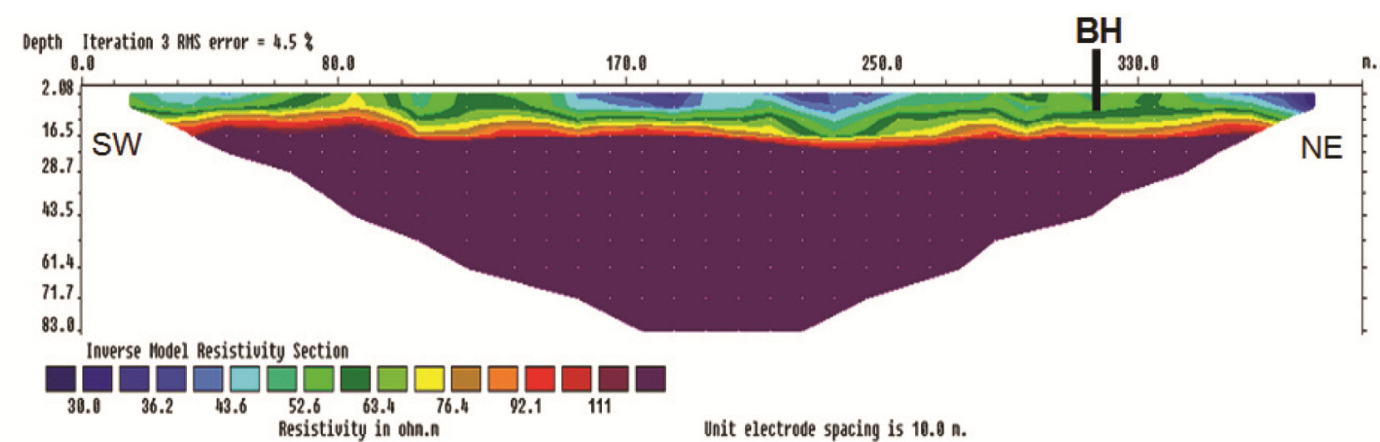

Figure 5. Mixed array 2D inverted resistivity model with changed scale of resistivity highlighting only the near-surface layer up to a depth of $\sim 20 \mathrm{~m}$ along with borehole location $(\mathrm{BH})$.

hand, Kumar et al. ${ }^{32}$ have also reported that the true resistivities from gradient array model corresponding to dipole-dipole array are smaller than those from gradient array in the same region. Although the broad geological structure is the same, the observed changes in resistivity values do not indicate any consistent relationship. Therefore, we have merged the datasets and processed to construct a composite model (Figure $4 c$ ). One can notice that the overall resistivity structure in the composite model is consistent with the geological regime of the region and also compatible with the resistivity models from individual array data. We can identify the shallow, lowresistivity zone with resistivity changes $<150 \mathrm{Ohm}-\mathrm{m}$ in the near-surface layer up to $\sim 20 \mathrm{~m}$ depth (Figure 5). This could be the weathered shallow aquifer zone. At depth $>20 \mathrm{~m}$, the formation resistivity increases with depth, which is inferred as semi-weathered/massive granite. As the main focus here is to discuss the shallow groundwater zones from the conventional as well as mixed/composite arrays, we have modified the resistivity scale to highlight 


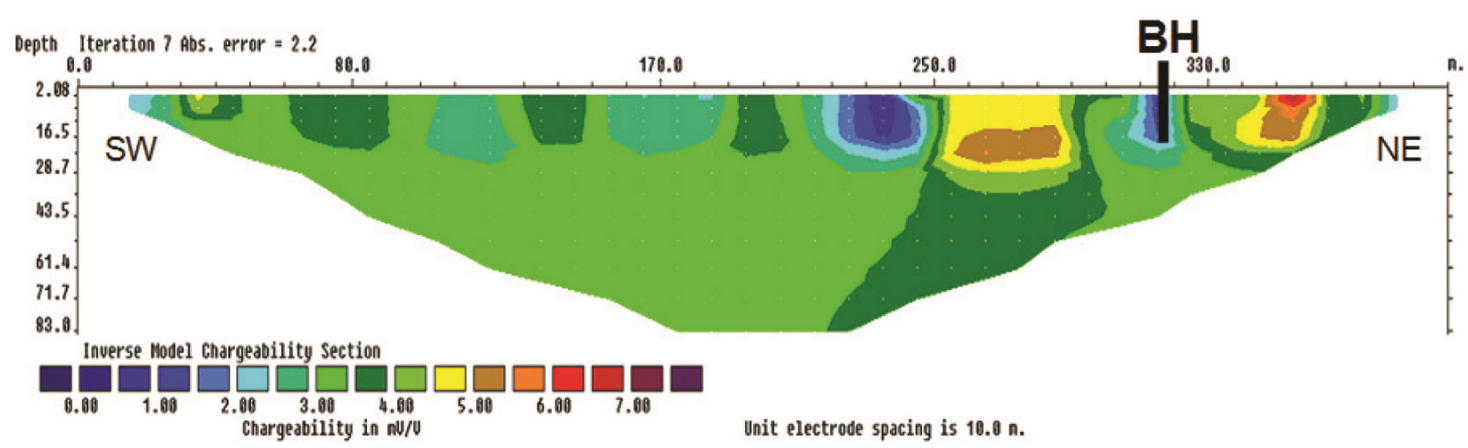

Figure 6. Two-dimensional inverted model chargeability using mixed array data along the resistivity profile with borehole location $(\mathrm{BH})$.

changes in the near surface up to 20-25 m (Figure 5). If we consider the mixed array $2 \mathrm{D}$ resistivity model with changed scale highlighting only the near-surface layer up to a depth of $\sim 20 \mathrm{~m}$ (Figure 5), it can be clearly seen that the resistivity varies from $<34$ to $150 \mathrm{Ohm}-\mathrm{m}$. This variation indicates weathered rock formation and moisture content within the conducting weathered formation composed of clay particles. It can be seen that the borehole location is connected well with the low-resistivity waterbearing $(<50 \mathrm{Ohm}-\mathrm{m})$ regions. The borehole drilled in the northeastern part of the area $(\mathrm{BH}$, Figure 5) shows that water level fluctuates between $7.8 \mathrm{~m}$ and $29 \mathrm{~m} \mathrm{bgl}$ during pre-monsoon to post-monsoon period. The borehole data also corroborate with resistivity variation of the weathered layer up to $20 \mathrm{~m}$ depth. The resistivity range $(<50 \mathrm{Ohm}-\mathrm{m})$ observed in the shallow portion of Figure 5 is within the range for fresh groundwater (10-100 Ohm-m), and suggests water saturation in shallow weathered layer.

Along with the resistivity models, the $2 \mathrm{D}$ inverted chargeability model was developed to study the variations in electrical conductivity of the near-surface geological formations up to a depth of $20 \mathrm{~m}$. The chargeability model shows a distinct variation in chargeability all along the section (Figure 5) and the variation is between close to zero and $7.0 \mathrm{mV} / \mathrm{V}$, which indicates the presence of different conducting materials as well as availability of groundwater. Very low chargeability (close to zero) noticed below the borehole location (Figure 6) confirms the presence of water bearing weathered aquifer. The effect of conducting minerals and rock materials is clearly seen up to a depth of $20 \mathrm{~m}$ (Figure 6).

\section{Conclusion}

The composite method, in which the apparent resistivity data from different electrode arrays were combined and inverted to produce a single resistivity model of the subsurface, brings out both lateral and depth wise resolution with optimal sensitivity and resolves the uncertainty in interpretation that arises due to the mismatch of resistivity models obtained using individual electrode arrays. From the physical model example, it is clear that the crosssections obtained from the composite dataset reflect the true target parameters. The target thickness estimation in case of thin resistive formations like marble sheets is also more appropriate in the composite model. As the composite data provide additional information about thin layers present in the subsurface than the individual arrays, this method is highly useful and sensitive for studying thin layers. The success of the borehole identified from the composite model of resistivity and chargeability of field data suggests the merit of the composite method. This procedure is applicable in $2 \mathrm{D}$ and $3 \mathrm{D}$ resistivity and IP studies to answer various geophysical and environmental problems.

1. Griffiths, D. H., Turnbull, J. and Olayinka, A. I., Two-dimensional resistivity mapping with a computer-controlled array. First Break, 1990, 8(4), 121-129.

2. Panissod, C., Dabas, M., Hesse, A., Jolivet, A., Tabbagh, J. and Tabbagh, A., Recent developments in shallow depth electrical and electrostatic prospecting using mobile arrays. Geophysics, 1998, 65, 1542-1550.

3. Loke, M. H. and Lane Jr, J. W., Inversion of data from electrical resistivity imaging surveys in water-covered areas. Explor. Geophys., 2004, 35, 266-271.

4. Loke, M. H., Tutorial: 2-D and 3-D electrical imaging surveys. 2001; http://www.geoelectrical.com (accessed on 13 March 2001).

5. Griffiths, D. H. and Barker, R. D., Two-dimensional resistivity imaging modeling in areas of complex geology. J. Appl. Geophys., 1993, 29, 211-226.

6. Loke, M. H. and Barker, R. D., Practical techniques for 3D resistivity surveys and data inversion. Geophys. Prospect., 1996, 44, 499-523.

7. Bhattacharya, P. K. and Patra, H. P., Direct Current Geo-electric Sounding, Principles and Interpretation, Elsevier, New York, USA, 1968.

8. Apparao, A., Reddy, B. S. and Sarma, V. S., Comparison of electrode arrays in induced polarisation and resistivity profiling. Geoviews, 1981, 9, 405-418.

9. Evjen, H. M., Depth factor and resolving power of electrical measurements. Geophysics, 1938, 3, 78-95.

10. Carpenter, E. W., Some notes concerning the Wenner configuration. Geophys. Prospect., 1955, 3, 388-402.

11. Carpenter, E. W. and Habberjam, G. M., A tri-potential method of resistivity prospecting. Geophysics, 1956, 21, 455-469. 


\section{RESEARCH ARTICLES}

12. Frohlich, R. K., The depth penetration of dipole arrays compared with Schlumberger arrangement. Geoexploration, 1967, 5, 195-204.

13. Apparao, A. and Roy, A., Resistivity model experiments. Geoexploration, 1971, 7, 45-54.

14. Apparao, A. and Roy, A., Resistivity model experiments II. Geoexploration, 1971, 9, 195-206.

15. Apparao, A. and Roy, A., Field results for direct current resistivity profiling with two-electrode array. Geoexploration, 1973, 11, 21-44.

16. Roy, A. and Apparao, A., Depth of investigation in direct current methods. Geophysics, 1971, 36(5), 943-959.

17. Roy, A., Depth of investigation in Wenner, three-electrode and dipole resistivity methods. Geophys. Prospect., 1972, 20, 329-340.

18. Roy, A. and Jain, S. C., Comparative field performance of electrode arrays in time-domain induced polarisation profiling. Geophys. Prospect., 1973, 42, 624-634.

19. Apparao, A., Srinivas, G. S. and Subrahmanya Sarma, V., Modelling results on modified pseudo-depth sections in exploration of highly resistive targets-II. $P A G E O P H, 1977, \mathbf{1 5 0}, 341-352$.

20. Apparao, A., Reddy, B. S. and Sarma, V. S., Model tank experiments on comparative performance of different electrode arrays in IP and resistivity profiling. In Symposium on Exploration Geophysics in India, 1945-78, Geological Survey of India, Calcutta, 1978.

21. Apparao, A. and Sarma, V. S., The modified pseudo-depth section as a tool in resistivity and IP prospecting - a case history. PAGEOPH, 1983, 121, 91-108.

22. Saydam, A. S. and Duckworth, K., Comparison of some electrode arrays for their IP and apparent resistivity responses over a sheetlike target. Geoexploration, 1978, 16, 267-291.

23. Sarma, V. S., Srinivas, G. S. and Joshi, M. S., Physical modeling results on modified pseudo-depth sections in exploration of highly resistive targets - II. PAGEOPH, 2001, 158, 813-820.

24. Oldenburg, D. W. and Li, Y., Estimating depth of investigation in DC resistivity and IP surveys. Geophysics, 1999, 64, 403-416.

25. Szalai, S., Novák, A. and Szarka, László, Depth of investigation and vertical resolution of geoelectric arrays. J. Environ. Eng. Geophys., 2009, 14(1), 15-23.
26. Lane Jr, J. W., Day-Lewis, F. D., Loke, M. H., Eric, A. and White, E. A., Pitfalls in inversion and interpretation of continuous resistivity profiling data - effects of resolution limitations and measurement error (abs.): EOS Trans., Am. Geophys. Union (Fall Meeting Suppl.), 2005, 86(52), abstract H43F-0546.

27. Patella, D., Introduction to ground surface self-potential tomography. Geophys. Prospect., 1997, 45, 653-681.

28. Mauriello, P., Monna, D. and Patella, D., 3D geoelectric tomography and archaeological applications. Geophys. Prospect., 1998, 46, 543-570.

29. Mauriello, P. and Patella, D., Resistivity anomaly imaging by probability tomography. Geophys. Prospect., 1999, 47, 411-429.

30. Barker, R. D., Depth of investigation of collinear symmetrical four-electrode arrays. Geophysics, 1989, 54(8), 1031-1037.

31. Rajesh, R., Padmavathi Devi, P., Sarma, V. S. and Rajendra Prasad, P., Electrical resistivity imaging over natural (in situ) geological samples using physical model studies. Arab. J. Geosci., 2013, 7(11), 4717-4725; doi:10.1007/s12517-013-1101-4.

32. Kumar, D. et al., Mapping lithology and assessing recharge characteristics in a granitic hard rock aquifer: inference from $2 \mathrm{D}$ resistivity, induced polarization, tracer and moisture measurements. J. Geol. Soc. India, 2016, 88(1), 29-38.

ACKNOWLEDGEMENTS. We thank Dr V. M. Tiwari, Director, CSIR-NGRI, Hyderabad for his encouragement towards the productive research and permission to publish this paper. We are grateful to CSIRNGRI for funding and necessary infrastructure. R.R. thanks CSIR, New Delhi for Research Associate fellowship. We are also thankful to the anonymous reviewers for their constructive comments, which improve the quality of this paper.

Received 12 January 2018; revised accepted 7 January 2019

doi: $10.18520 / \mathrm{cs} / \mathrm{v} 116 / \mathrm{i} / 1356-1362$ 